EUROPEAN ORGANIZATION FOR NUCLEAR RESEARCH

European Laboratory for Particle Physics

Large Hadron Collider Project

LHC Project Report 511

\title{
QUENCH PROPAGATION AND HEATING \\ IN THE SUPERCONDUCTING 600 A AUXILIARY BUSBARS OF THE LHC
}

R. Herzog, M. Calvi and F. Sonnemann

\begin{abstract}
In the Large Hadron Collider (LHC) at CERN $22 \mathrm{~km}$ of flexible superconducting cable, the auxiliary busbar cable, will conduct currents of up to $600 \mathrm{~A}$ to a large number of corrector magnets distributed throughout the accelerator. A prototype cable with 42 active conductors underwent several experiments to measure the hot spot temperature and the quench propagation velocity as a function of the current. The former was evaluated for various energy extraction scenarios as they are foreseen for the LHC corrector circuits.

The experimental results and the heat flow simulations show that the quench behavior in this busbar prototype is strongly influenced by the heat flow through the insulation material (polyimide) into the helium bath, leading to stable configurations above the critical temperature $T_{c}$ for currents between 250 $\mathrm{A}$ and $500 \mathrm{~A}$.

Special attention was paid to the study of discontinuities in the wires, like feed-throughs, where the wire is not immersed in liquid helium, and joints, where the wire cross-section is increased.

The experiments and simulations led to a thorough understanding of the quench process in the wires of the prototype cable, which resulted in guidelines for the design, the use and the installation of the cable in the LHC.
\end{abstract}

LHC Division

Presented at the 2001 Cryogenic Engineering Conference and International Cryogenic Materials Conference CEC/ICMC 2001

16-20 July 2001, Madison, Wisconsin, USA

Administrative Secretariat

LHC Division

CERN

CH - 1211 Geneva 23

Switzerland

Geneva, 19 October 2001 


\title{
QUENCH PROPAGATION AND HEATING IN THE SUPERCONDUCTING 600 A AUXILIARY BUSBARS OF THE LHC
}

\author{
R. Herzog, M. Calvi and F. Sonnemann \\ CERN, LHC Division, 1211 Geneva 23, Switzerland
}

\begin{abstract}
In the Large Hadron Collider (LHC) at CERN $22 \mathrm{~km}$ of flexible superconducting cable, the auxiliary busbar cable, will conduct currents of up to $600 \mathrm{~A}$ to a large number of corrector magnets distributed throughout the accelerator. A prototype cable with 42 active conductors underwent several experiments to measure the hot spot temperature and the quench propagation velocity as a function of the current. The former was evaluated for various energy extraction scenarios as they are foreseen for the LHC corrector circuits.

The experimental results and the heat flow simulations show that the quench behavior in this busbar prototype is strongly influenced by the heat flow through the insulation material (polyimide) into the helium bath, leading to stable configurations above the critical temperature $T_{\mathrm{c}}$ for currents between $250 \mathrm{~A}$ and $500 \mathrm{~A}$.

Special attention was paid to the study of discontinuities in the wires, like feedthroughs, where the wire is not immersed in liquid helium, and joints, where the wire crosssection is increased.

The experiments and simulations led to a thorough understanding of the quench process in the wires of the prototype cable, which resulted in guidelines for the design, the use and the installation of the cable in the LHC.
\end{abstract}

\section{INTRODUCTION}

For the tuning of LHC beam parameters and the correction of field errors in the main magnets, about 6000 corrector magnets with currents up to $600 \mathrm{~A}$ will be installed along a large part of the $27 \mathrm{~km}$ circumference of the accelerator. Because power converters and current leads can only be located at both ends of the eight octants, about $800 \mathrm{~km}$ of superconducting wire are required to connect the magnets to the converters [1]. For the supply of the corrector magnets close to the main quadrupoles, $53 \mathrm{~m}$ long segments of a 42 wire cable will be installed in a tube which is welded to the helium pressure vessel of the main magnets and filled with $1.9 \mathrm{~K}$ helium. This busbar layout was conceived to minimize the number of joints, potential sources of error, and has been slightly modified since [1] to reflect recent changes in the powering scheme and beam optics of the LHC. 
TABLE 1. Required cable properties

\begin{tabular}{ll|ll}
\hline Parameter & Value & Parameter & Value \\
\hline Number of conductors & 42 & Copper cross section of one wire & $1.8 \mathrm{~mm}^{2}$ \\
Maximal current per conductor at $1.9 \mathrm{~K}$ & $600 \mathrm{~A}$ & Minimum RRR of copper & 100 \\
Min. critical current at $1.9 \mathrm{~K}$ and $50 \mathrm{mT}$ & $1 \mathrm{kA}$ & Insulation withstand voltage & $3 \mathrm{kV}$ \\
Minimum bending radius of cable & $200 \mathrm{~mm}$ & Radiation dose the cable has to withstand & $60 \mathrm{kGy}$ \\
\hline
\end{tabular}

Practical tests showed that the pulling of $53 \mathrm{~m}$ long superconducting cables into ducts is feasible and that the foreseen expansion loops are capable of compensating the $0.35 \%$ thermal contraction of the cable, during the cool-down to $1.9 \mathrm{~K}$. The cable however, needs to be rather flexible for installation. Electrical tests on a first prototype showed that the operation of the cable at nominal conditions (in $1.9 \mathrm{~K}$ helium and with currents up to $600 \mathrm{~A}$ ) does not pose any problems [1], if the conductors are held well in place inside the cable. Otherwise conductor movement might trigger a quench, which is undesirable for the operation of the LHC. The apparently contradictory quests for a flexible yet also firm cable could be solved by the design described below.

If a quench occurs, it is essential that no part of the superconducting circuit is damaged. Quench events need to be detected and the current in the circuit reduced to zero in a controlled manner, before the maximum temperature in the circuit, usually appearing at the quench origin, reaches excessive levels. The components of a superconducting circuit must be dimensioned to cope with its specific conditions of quench detection and energy extraction. The following describes the methods used to achieve a design of the auxiliary busbar cable for the LHC that can be adequately protected.

\section{DESIGN OF THE SECOND PROTOTYPE CABLE}

Recent changes of the LHC optics layout [2] allowed for the reduction of the number of wires in line $\mathrm{N}$ to 42 , which can all be accommodated in a single 3-layer cable. Other required cable properties are summarized in TABLE 1. Improving on the design of the first busbar cable [1], a cable manufacturer produced a second prototype with the cross section shown in FIG 1. The central former is made of a multifilamentary copper wire. The three layers of 8,14 and 20 superconducting wires have pitch lengths of 74, 107 and $137 \mathrm{~mm}$

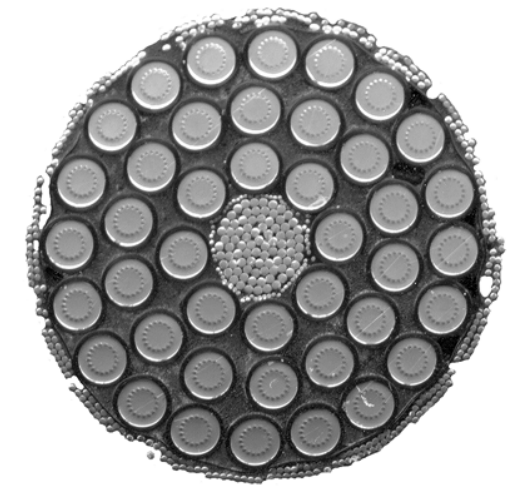

FIGURE 1. Cross section of the second prototype cable. Center: multifilamentary copper former; three layers of superconducting wires, laid in alternating senses; polyethylene bedding tape and on top a protective stainless steel braid. Overall diameter: $16.4 \mathrm{~mm}$. respectively, which is close to the technical minimum of the cable winding process. Each wire is insulated with two layers of wrapped polyimide film for a total insulation thickness of $0.2 \mathrm{~mm}$. The short pitch lengths ensure a flexible cable, yet the wires inside are kept well in place, because a prestress from the manufacturing process results in a force pushing the wires towards the center of the cable. A polyester bedding tape was wound on top of the third layer to protect it from the final stainless steel braid, which in turn protects the cable from external impacts. The braid also creates an additional force to keep the cable together.

One of the key parameters with which to influence the quench behavior in busbars is the cross section of the copper stabilization $A_{\mathrm{Cu}}$. The value chosen for the prototype cables was derived from two assumptions: The worst case occurs at the maximal operating current $I_{\max }$ because the stored energy is largest and the extrac- 
tion time is longest. The quench detection time was calculated from a realistic detection voltage of $0.3 \mathrm{~V}$ and an estimate of the quench propagation velocity at $I_{\max }$. The second assumption was adiabatic behavior of the quenched wire, which leads to a direct relationship between $\int I^{2}(t) d t$, the so called quench load, and the maximal wire temperature at the end of the energy extraction [3]. The integral is evaluated from the start of the quench until the current in the wire is zero.

Although there was confidence that the resulting wire cross section is suitable at maximal current $(600 \mathrm{~A})$, it was not clear that a quench at lower currents could not possibly lead to a situation where cooling of the wire by the surrounding helium would substantially reduce the spreading of the quenched zone and hence increase the detection time, during which the hot spot temperature might rise to unacceptable levels. To ensure the safe deexcitation of all LHC circuits at all currents, a better understanding of the quench process was needed. This was achieved with simulations using a more realistic electro-thermal model and complementary quench propagation experiments on the prototype cable.

\section{QUENCH SIMULATIONS}

The symmetry of a round wire suggests the use of cylindrical coordinates. Often it is reasonable to assume all variables to be independent of $r$ (e.g. constant temperature over the copper cross-section), such that the problem reduces to a one-dimensional one along the wire axis. The heat balance equation for this case becomes:

$$
\frac{d}{d z}\left(k(T(z, t)) \frac{d T(z, t)}{d z} A(z)\right)-h^{\mathrm{He}}(T(z, t), t) P(z)+G(T(z, t), t)=c(T(z, t)) A(z) \frac{d T(z, t)}{d t}
$$

The first term describes the heat flow along the wire in the metal (weighted average of copper and $\mathrm{NbTi}$ ), which is influenced by the heat conductivity of the metal $k(T)$ and a possibly changing wire cross section $A(z)$. The second term takes the heat flow through the insulation into the helium bath into account, weighted with the wetted perimeter $P(z)$, and the third term describes the heat generation by ohmic heating, which is explicitly time dependent for varying currents. The net heat generation in a wire element leads to a temperature increase per unit time which is inversely proportional to the heat capacity $c(T)$. $G$ is 0 nearly up to $T_{\mathrm{c}}$ and $\rho(T(x, t)) I^{2}(t) / A(z)$ above $T_{\mathrm{c}}$ with a narrow transition in between [3]. From the operating temperature of $1.9 \mathrm{~K}$ up to room temperature, the three material parameters $k(T), c(T)$ and $\rho(T)$ vary substantially, and an analytical solution of equation (1) for $T(z, t)$ is therefore impossible to obtain. The equation was therefore discretized and time integrated with an implicit finite difference method [4,5] by using a specially written $\mathrm{C}$ program. A Gaussian temperature profile at the origin served as the initial condition for the quench simulations.

\section{Heat Transfer Through The Insulation Layer}

Materials used for electrical insulation, like the polyimide of our prototype cables, are often poor thermal conductors, especially so at cryogenic temperatures. But although the heat flow from a heating-up conductor into the helium bath is limited, it is decisive to describe the quench propagation realistically. It was therefore calculated for quasi stationary problems and problems with a fast temperature rise to enter as $h^{\mathrm{He}}(T, d T / d t)$ into equation (1).

Neglecting the heat flow in longitudinal direction (along $z$ ) inside the insulation, the radial heat flow follows the heat balance equation in cylindrical coordinates 


$$
\frac{1}{r} \frac{d}{d r}\left(r k_{\mathrm{ins}}\left(T_{\mathrm{ins}}\right) \frac{d T_{\mathrm{ins}}}{d r}\right)=c_{\mathrm{ins}}\left(T_{\mathrm{ins}}\right) \frac{d T_{\mathrm{ins}}}{d t}
$$

In the stationary case the right hand side is zero and the expression in brackets, the total heat flow through a cylinder per unit length with radius $r$, is independent of $r$ (constant). For a given temperature at the boundary between the copper surface of the wire and the insulation $T_{\mathrm{Cu}}$, a functional relation between the heat flow and the surface temperature of the insulation $T_{\text {ins }}$ can be found by numerical integration of equation (2) ( $F_{1}$ in FIG 2).

At the surface of the insulation the heat flow into the helium bath is governed by several mechanisms, including, for superfluid helium and heat flows up to $100 \mathrm{~kW} / \mathrm{m}^{2}$, the Kapitza conductance $[3,6]$. Usually there is a one-to-one relationship between the surface temperature $T_{\text {ins }}$ and the heat flow, but at the transition from nucleate boiling to film boiling, the heat flow reduces sharply with $T_{\text {ins. }}$. A simplified functional dependence between $T_{\text {ins }}$ and the heat flow into the helium bath is plotted as a bold line in FIG $2\left(F_{2}\right)$.

With this relation and the solution of equation (2), it is possible to calculate the stationary heat flow and $T_{\text {ins }}$ for every $T_{\mathrm{Cu}}$ from the intersection points of the functions $F_{1}$ and $F_{2}$ in FIG 2. It is noteworthy that for $T_{\mathrm{Cu}}$ up to $180 \mathrm{~K}, T_{\mathrm{ins}}$ remains below $5 \mathrm{~K}$, a consequence of the excellent transfer of heat into helium on the insulation surface in the Kapitza regime and the poor thermal conductivity of the insulation itself. FIG 3 shows typical temperature profiles inside the insulation. The heat flow from the metal wire is thus drastically limited by the insulation in comparison to a bare wire in superfluid helium.

If the wire temperature increases slowly $(d T / d t \ll 100 \mathrm{~K} / \mathrm{s})$, the stationary approach is adequate, but in situations with $d T / d t>100 \mathrm{~K} / \mathrm{s}$, as they occur at the front line of an expanding normal zone, the heat capacity of the insulation material can lead to an increased heat flow out of the metal wire. To remain with the one-dimensional model of equation (1), this effect was taken into account by calculating the heat flow from the wire into the insulation in advance for an array of wire temperatures and changes of wire temperatures $(d T / d t)$. Equation (2) was solved numerically with a finite difference method and a series of $T_{\mathrm{Cu}}(t)$ as boundary condition to obtain the function $h^{\mathrm{He}}(T, d T / d t)$ tabulated in a two dimensional array.

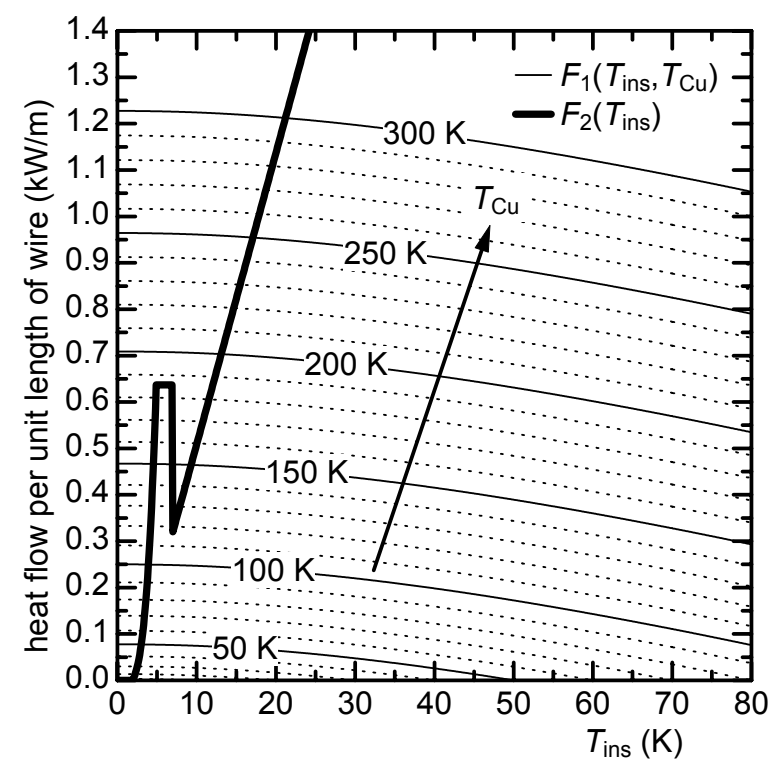

FIGURE 2. Heat flow through the insulation as a function of $T_{\text {ins }}$ and $T_{\mathrm{Cu}}$ (thin lines) and heat flow from the insulation into the helium as a function of $T_{\text {ins }}$ (bold line). The radius of the bare wire is $0.8 \mathrm{~mm}$; the insulation thickness $0.2 \mathrm{~mm}$.

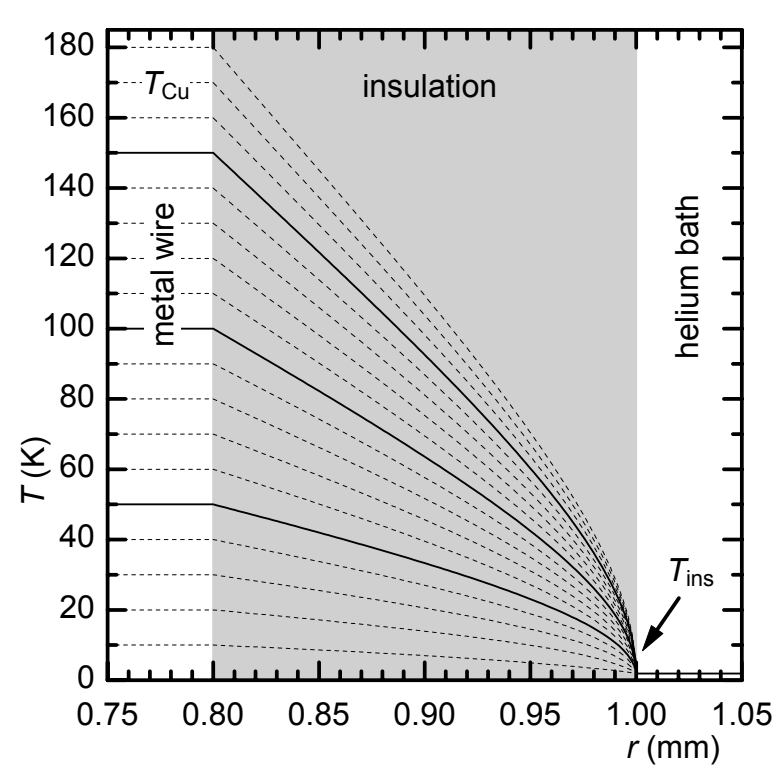

FIGURE 3. Temperature profiles in the insulation for constant wire temperatures $T_{\mathrm{Cu}}$. These are solutions for stationary heat flow through the insulation. 


\section{Simulation Results}

Simulations with the parameters of the prototype cable described above revealed a number of noticeable features over the whole range of operating currents. Below a current $I_{0}$ of about $250 \mathrm{~A}$, quenches did not propagate, even for fairly large and 'hot' initial temperature profiles $\left(T_{\max }<30 \mathrm{~K}\right)$. Above this current up to $I_{1}(\approx 510 \mathrm{~A})$, quenches do propagate with ever increasing velocity, but the temperature at the quench origin remains limited to values below $30 \mathrm{~K}$.

Looking at the balance of the heat flow through the insulation (cooling power) and heating power at different currents, it is possible to explain this behavior (FIG 4). Below $175 \mathrm{~A}$, the cooling power is larger than the heating power for all wire temperatures, and thus an initial quench will always be cooled away. For currents between 200 and $510 \mathrm{~A}$, a temperature region exists ranging from $T_{\mathrm{c}}$ up to $T_{\mathrm{s}}$ where heating is stronger than cooling, followed by a region where cooling is again stronger than heating. Wire parts heated to temperatures above $T_{\mathrm{c}}$ in the course of a quench will either be heated or cooled to the boundary temperature $T_{\mathrm{s}}$ of the two regions, which is a stable equilibrium point (marked with a dot $(\bullet)$ for the 400 A heating curve). Once the 'inner' parts of a normal zone are at $T_{\mathrm{s}}$, which happens relatively quickly, the zone either expands, shrinks or remains stationary. For the last case, the right hand side of equation (1) is zero, and a solution only exists for the current $\left(I_{0}\right)$ at which the equal area theorem is fulfilled [7]. The theorem states that at $I_{0}$ the two left shaded areas in FIG 4a are equal. Towards the ends of the normal zone, the extra heat which is generated in the wire section with a $T$ between $T_{\mathrm{c}}$ and $T_{\mathrm{s}}$ flows along the wire and eventually into the helium bath in the section with a $T$ between $T_{\text {bath }}$ and $T_{\mathrm{c}}$. If the heat generation between $T_{\mathrm{c}}$ and $T_{\mathrm{s}}$ is larger than the cooling between $T_{\mathrm{bath}}$ and $T_{\mathrm{c}}\left(I>I_{0}\right)$, the normal zone expands; if heat generation is smaller, the zone shrinks $\left(I<I_{0}\right)$.

Above $I_{1}$, heating is stronger than cooling for all $T$ above $T_{\mathrm{c}}$, and no stable equilibrium between cooling and heating exists in the normal state of the wire. For quenches at $I>I_{1}$, the hot-spot temperature increases continuously and particularly fast when $T$ exceeds $50 \mathrm{~K}$, because the increasing resistivity of the copper is not matched by a comparable increase of cooling into the helium bath.

The temperature dependent materials parameters lead directly to the curves in FIG 4

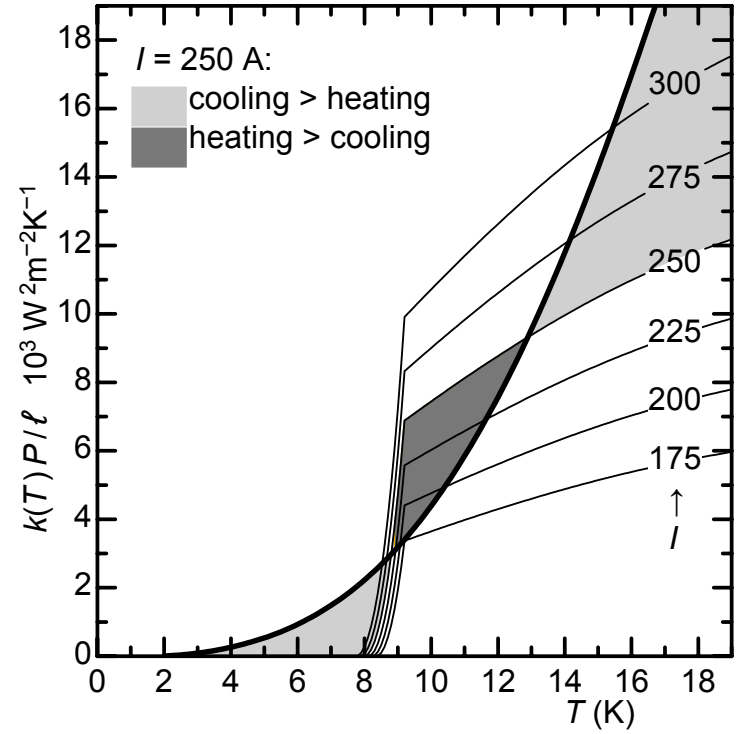

FIGURE 4a. Cooling and heating power per unit length of wire, both multiplied with the copper heat conductivity $k(T)$, to directly show the equal area theorem for $I_{0}=250 \mathrm{~A}$.

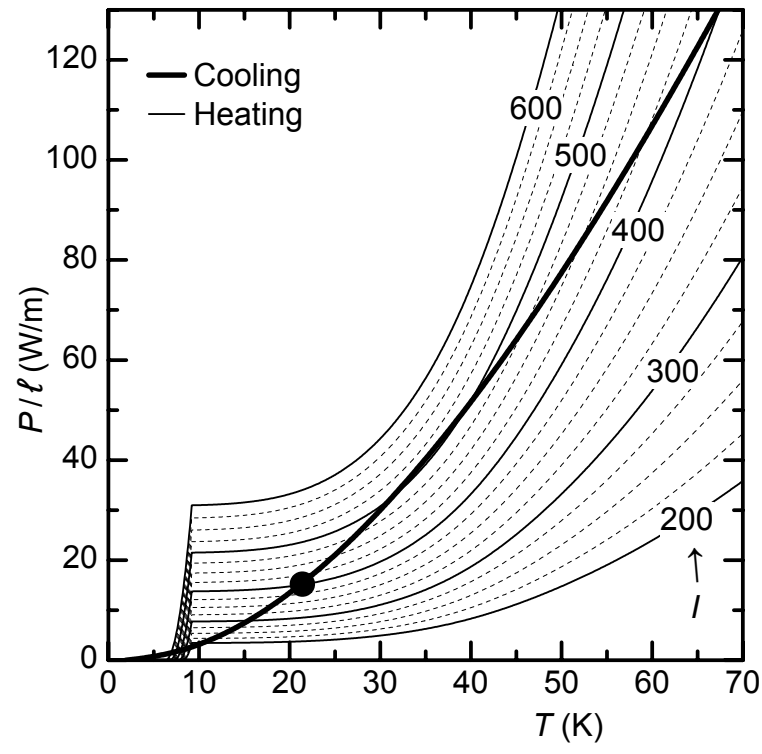

FIGURE 4b. Cooling and heating power per unit length of wire for higher currents and temperatures. For currents above 510 A only one crossing point between cooling and heating curves exists close to $T_{\mathrm{c}}$. 
and, with the considerations above, to the important parameters $I_{0}$ and $I_{1}$. To obtain the quench propagation velocity $v_{\mathrm{q}}(I)$ and $T_{\max }$ at any desired time a full simulation of the quench process, as outlined above, is necessary. FIG 5 shows the calculated $v_{\mathrm{q}}(I)$ together with experimentally measured values.

\section{QUENCH PROPAGATION EXPERIMENTS}

Measurements of the quench propagation velocity with a number of setups served as a test for the physical model at the basis of the simulations outlined above.

For the experiments, voltage taps were soldered to wire sections with lengths up to $6 \mathrm{~m}$ and small heaters were wrapped around the insulated wire at one location to trigger quenches. In one setup, the wire was left in its environment inside the cable, where it was covered with the bedding tape and the stainless steel braid. In another setup, the insulated wire was mounted in a zigzag shape on an insulated plate, but the obtained results are almost identical. The assemblies were installed in a $500 \ell$ cryostat operating at 1.9 or $4.2 \mathrm{~K}$. A 32-channel data logger recorded the voltages between adjacent taps at frequencies up to $10 \mathrm{kHz}$. A LHC prototype power converter supplied currents up to $1 \mathrm{kA}$, and quenches were triggered by discharging a large capacitor into the heater.

The quench propagation velocity was calculated from the voltage traces with a 'time of flight' method, where the length between two voltage taps is divided by the time the front line of the normal zone needs to pass from one tap to the other (onset of voltage). The measured velocities are in very good agreement with the results obtained with the simulations, for which materials parameters from the literature were used [8]. The experiments also confirmed the existence of stable thermal equilibria at $T$ between $T_{\mathrm{c}}$ and $30 \mathrm{~K}$ for currents between $I_{0}$ and $I_{1}$. The experiments confirmed the validity of the model on which the simulations are based, making them a useful tool for the evaluation of large scale designs, for which full scale tests are impractical.

\section{QUENCH PROPAGATION AT DISCONTINUITIES}

At the ends of the $53 \mathrm{~m}$ long cable sections the wires will pass through a helium-tight feedthrough made of polyimide (Vespel) and they will be joined to wires leading to

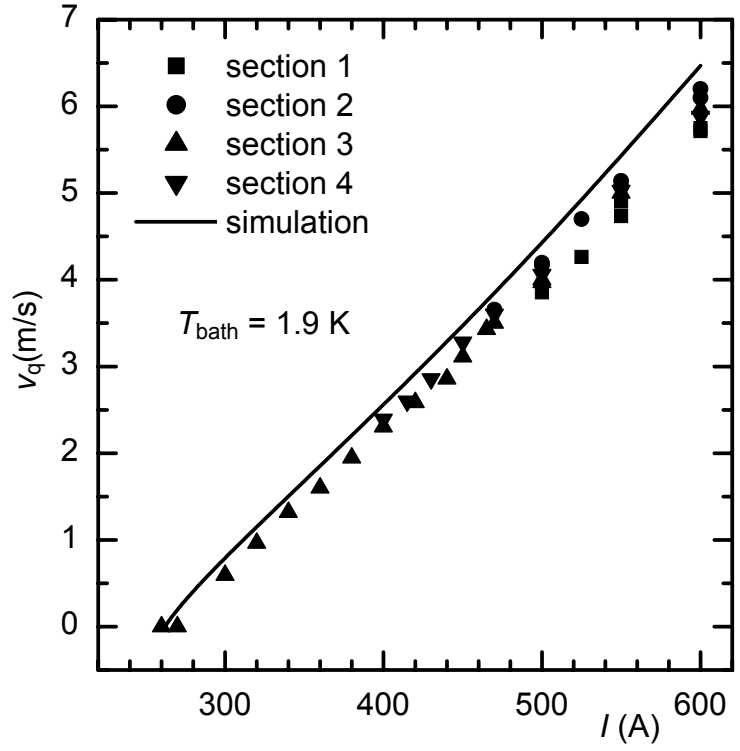

FIGURE 5. Quench propagation velocity as a function of current.

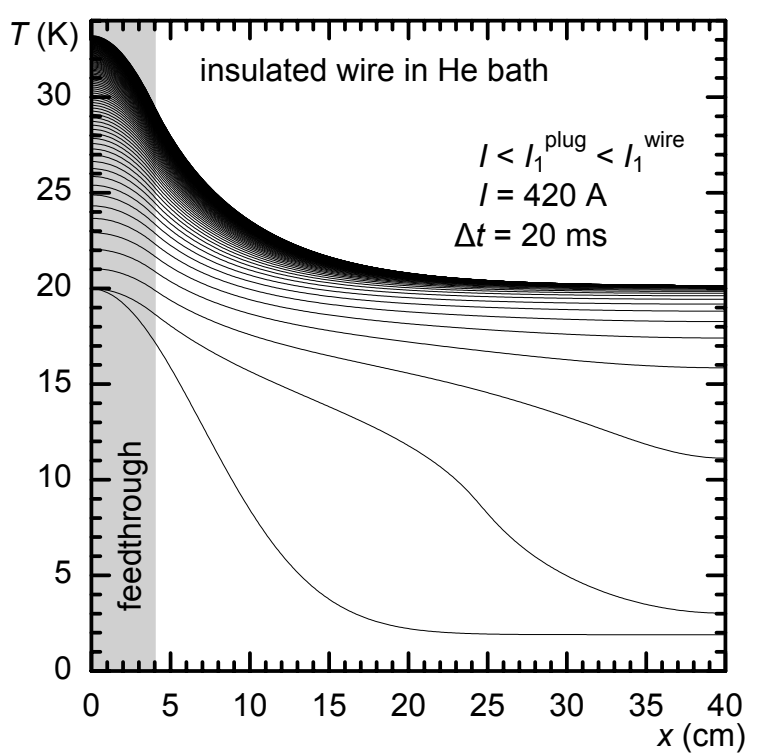

FIGURE 6. Simulation of the time development of the temperature profile inside and adjacent to a feedthrough. 
corrector magnets, as well as to the wires of the next cable section. At the feedthroughs the wires are less well cooled than in the helium bath, potentially leading to a situation where a quench occurring inside the feedthrough does not propagate out of it, but the hot-spot heats up in an uncontrolled way. At joints, on the other hand, the cross-section of the wire is doubled (less heating), and if the copper is in direct contact with the helium bath, the cooling is much increased. Joints are thus potential 'quench stoppers'.

Experiments on proposed feedthrough designs and simulations with their attributes reveal that their main effect is a reduction of $I_{1}$, but for reasonable lengths (up to $80 \mathrm{~mm}$ ) it remains well above the value of $I_{0}$ outside the feedthrough, even if the heat flow into the feedthrough material is set to zero. The reason for this behavior is that the heat generated in the wire inside the feedthrough can flow along the wire and eventually into the two adjacent helium baths. FIG 6 shows a typical simulation at 420 A, and FIG 7 shows the experimentally obtained $I_{1}$ values for several feedthrough lengths.

Joints insulated like the wire delay the propagation of a normal zone, with longer joints causing bigger delays, and they increase $I_{0}$. FIG 8 shows the front line of a normal zone (defined by the position where $T=T_{\mathrm{c}}$ ) advancing across three joints of different length. If a joint is not insulated, the properties change drastically. During the experiments, quenches could not propagate across $50 \mathrm{~mm}$ long soldered joints, even at $600 \mathrm{~A}$. Simulations show that $I_{0}$ increases to $500 \mathrm{~A}$ even for the $10 \mathrm{~mm}$ long joints made by ultrasonic welding as they are foreseen for the LHC.

\section{PROTECTION OF THE LINE N BUSBARS}

The protection system of a superconducting circuit needs to ensure that a quench occurring in any part of the circuit is detected and the current reduced to zero before any damage is caused. Therefore, both the quench detection time and the current extraction time should be as short as possible within practical constraints, like the minimum signal-to-noise ratio of the detection threshold voltage and the maximally acceptable circuit to ground voltage (discharge of inductive circuit).

With a current just above $I_{0}$, the normal zone in the busbar described above needs to be $16 \mathrm{~m}$ long to generate a voltage drop of $0.3 \mathrm{~V}$, a typical detection level foreseen for the

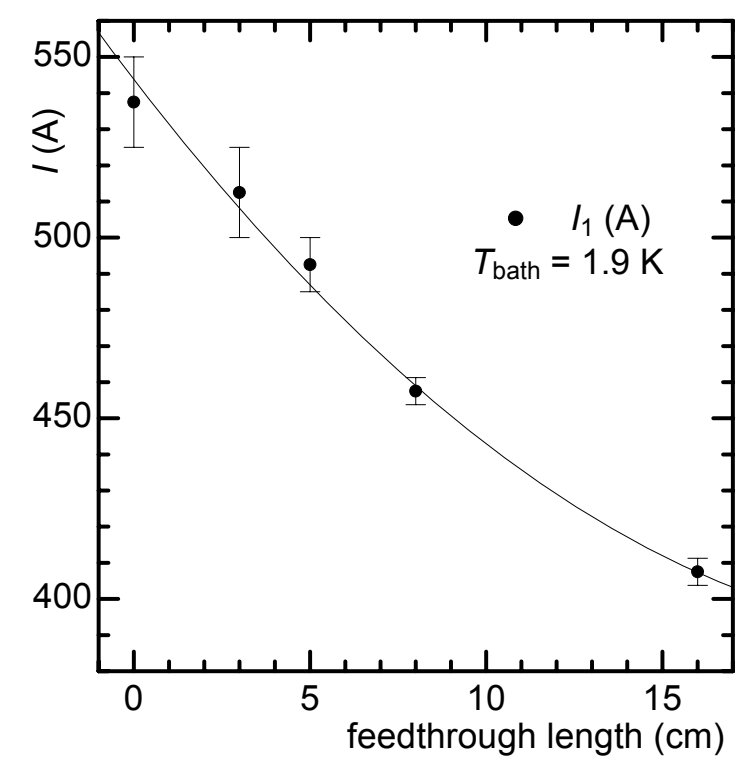

FIGURE 7. The current $I_{1}$ decreases with increasing feedthrough length, but stays well above $I_{0}$ of the wire in superfluid helium.

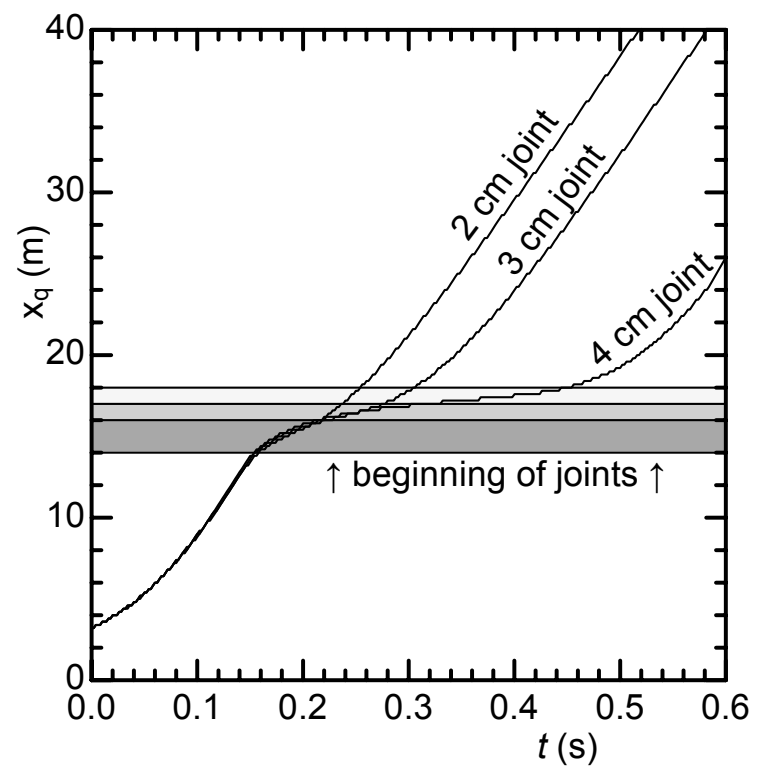

FIGURE 8. Simulation of a front line of a normal zone passing 2, 3 and $4 \mathrm{~cm}$ long joints, which are insulated with $0.2 \mathrm{~mm}$ polyimide. At $280 \mathrm{~A}$ the current is only slightly above $I_{0}$ 
LHC. A quench, where the quenched section is shorter than this length and cannot further expand because of adjacent quench stoppers, will not be detected. This does not lead to any damage, because $T_{\max }$ remains smaller than $30 \mathrm{~K}$, but the additional heat load from the resistive zone on the cryogenic system should be avoided.

At currents above $I_{1}$, the detection time is short, but the hot-spot temperature increases very quickly as well. One example where the simulation of the busbar helped with a design decision was the 56 circuits of the individually powered long trim quadrupoles (MQTLs). The wish to save a separate extraction resistor limited the extraction voltage to $30 \mathrm{~V}$, the maximum value at which the power converter can discharge the circuit. In case a quench occurs in the busbar, which does not reach the magnet, the resulting extraction time constant is $2.5 \mathrm{~s}$, during which time $T_{\max }$ can reach $300 \mathrm{~K}$. With a time constant only half a second longer, for example, $T_{\max }$ would go up to $450 \mathrm{~K}$, which is already unacceptable because of the glue used with the insulation. With the result from the busbar simulation, it was possible to accept energy extraction of the individually powered MQTL magnets by the power converters [9].

\section{CONCLUSIONS AND IMPLICATIONS}

The quench simulations and the experiments led to a thorough understanding of the quench process at all currents in the prototypes of the LHC auxiliary busbar cable. It became clear that the heat flow into the helium bath is limited by the heat transfer through the polyimide insulation, which is nevertheless decisive to adequately describe the quench propagation process $\left(I_{0}, I_{1}, v_{\mathrm{q}}\right)$. The detailed model developed for the heat flow through the insulation also led to quantitative agreement with the measurement results.

For the design of the superconducting circuits of the LHC, it became clear that short stretches of wire between 'quench stoppers' have to be avoided in order to ensure timely quench detection. Ideally quenches in busbars close to magnets should propagate into the magnet coils and trigger a quench there, because their resistance rises quickly when quenched, leading to easily detectable voltage levels. For the same reason, 'quench stoppers' should be avoided, and thus joints should be well insulated and no blank wire parts left uncovered.

The prototype cable evaluated here fulfilled all requirements, and its design can be recommended for the cable which will be deployed in the LHC.

\section{REFERENCES}

1. Herzog R., Dahlerup-Petersen K., Parente C., Schmidt R., Sonnemann F. and Teng M., "The Superconducting Busbar Cable Powering the Auxiliary Magnets of the LHC" in Advances in Cryogenic Engineering 45B, edited by Q.S. Shu et al., Kluwer Academic/Plenum, New York, 2000, pp. 1581-1588.

2. P. Proudlock, "Update on the Powering Strategy of LHC", Particle Accelerator Conference 1999, 29 April-2 May, New York, USA.

3. Wilson M. N., Superconducting Magnets, Oxford University Press, Oxford, 1983, p. 107, p. 94.

4. Sonnemann F. and Schmidt R., "Modelling of the Quench Process for the Optimization of the Design and Protection of Superconducting Busbars for the LHC" 18th International Cryogenic Engineering Conference edited by K. G. Narayankhedkar, Mumbai, 2000, pp. 183-186.

5. Press W. H., Flannery B.P., Teukolsky S. A., Vetterling W.T., Numerical Recipes in C, Cambridge University Press, Cambridge, 1993, chapter 19.2.

6. Sonnemann, F. and Schmidt, R., Cryogenics 40, pp. 519-529 (2000).

7. Maddock, B.J., James, G.B. and Norris, W.T, Crogenics Aug., p. 261 (1969).

8. HEPAK and CPPAK reference databases from Cryodata Inc. (www.cryodata.com).

9. Dahlerup-Petersen K., Rodriguez-Mateos F., Schmidt R. and Sonnemann F., "Energy Extraction for the LHC Superconducting Magnets" to be published in the proceedings of the Particle Accelerator Conference 2001. 